Noman 2017, 35(1), 31-38

Revista de Psicologia, Ciències de l'Educació i de l'Esport

ISSN: 1138-3194

Copyright (C) 2017

www.revistaaloma.net

\title{
Efecto terapéutico del afecto positivo
}

\author{
Fernando Gordillo-León ${ }^{1}$, Lilia Mestas-Hernández², Miguel Ángel Pérez-Nieto', \\ José M. Arana-Martínez ${ }^{3}$ \& Rafael Manuel López-Pérez ${ }^{4}$ \\ ${ }^{1}$ Universidad Camilo José Cela (UCJC) \\ ${ }^{2}$ Facultad de Estudios Superiores Zaragoza (UNAM, México D.F.) \\ ${ }^{3}$ Universidad de Salamanca \\ ${ }^{4}$ Fundación Universitaria Behavior \& Law
}

Recibido: 19-11-2016

Aceptado: 6-2-2017

\section{Efecto terapéutico del afecto positivo}

Resumen. La capacidad para regular las emociones de manera eficaz facilita la adaptación de las personas al ámbito social y es uno de los elementos fundamentales para entender cómo las personas más resilientes son capaces de afrontar las situaciones adversas y salir reforzadas. El análisis de estas variables permitiría comprender mejor la relación que la literatura científica establece entre el estado afectivo positivo y una mejor salud física y mental. Uno de los aspectos más relevantes en esta relación son las habilidades para reconocer y expresar las emociones, que permiten su fácil integración en programas de prevención y rehabilitación. Además, estas habilidades podrían potenciarse en el contexto clínico a través de la mejora en la interacción emocional entre el personal sanitario y los pacientes, con un enfoque prioritario hacia la regulación de las emociones positivas.

Palabras clave: amígdala; emoción; expresión facial; regulación emocional

The therapeutic effect of positive affect

Summary. The ability to regulate emotions effectively makes it easier for individuals to adapt to their social context, and it is one of the keys to understanding how the most resilient people are better able to cope with adverse situations and emerge from them stronger than before. An analysis of these variables would allow for a better understanding of the relationship that previous scientific literature has established between positive emotional states and improved physical and mental health. Among the most important aspects of this relationship is an ability to recognize and express emotions, which would indicate that these skills would be suitable for inclusion in prevention and rehabilitation programs. In addition, these abilities can be enhanced in the clinical context through improved emotional interaction between health professionals and patients, aimed at fostering positive emotion regulation.

Key words: amygdala; emotion; facial expression; emotional regulation 


\section{Introducción}

El estado afectivo positivo se ha relacionado con una mejor salud física y mental, pero no queda claro que esta relación pueda explicarse por su capacidad para promover cogniciones y comportamientos saludables (Cameron, Bertenshaw \& Sheeran, 2014). Sin embargo, las personas que manifiestan ser más felices tienen mejor salud mental y física; menos trastornos como la depresión, la ansiedad, la hipocondriasis o la esquizofrenia; menor probabilidad de consumir drogas, delinquir o tener reacciones alérgicas; visitan menos los hospitales, toman menos medicamentos, piden menos bajas laborales por problemas de salud y tienen una mejor calidad de vida en enfermedades como el cáncer (Lyubomirsky, King \& Diener, 2005). A la luz de estos datos, las estrategias de intervención centradas en la regulación de las emociones positivas, tanto de carácter preventivo como dentro de los tratamientos de diferentes enfermedades y trastornos mentales, podrían tener efectos beneficiosos a corto y largo plazo. La presente revisión tiene por objetivo analizar el papel de las emociones en los procesos de adaptación al medio. En un primer momento se establecerá de manera clara la relación entre las emociones positivas y los procesos de adaptación (regulación emocional y resiliencia), y, posteriormente, se discutirá la posibilidad de utilizar esta relación en el ámbito clínico como instrumento terapéutico.

En la psicología positiva se considera que el ser humano tiene la capacidad de adaptarse y recuperarse de situaciones adversas (Vera, 2006), pero no todas las personas pueden salir reforzadas de una situación desfavorable, por lo que resulta de especial relevancia saber qué variables estarían determinando estas diferencias. Una de estas variables es la resiliencia o capacidad que manifiestan algunas personas para gestionar de manera adecuada y adaptativa las funciones físicas y psicológicas en situaciones críticas. Se puede entender como un proceso dinámico que varía atendiendo a las circunstancias, la naturaleza de la situación, el contexto y la etapa de la vida (Carretero, 2010). De manera más específica, el rasgo de resiliencia se ha relacionado con una mejor salud mental (Hu, Zhang \& Wang, 2015), por lo tanto, facilitaría el uso de estrategias de afronta- miento eficaces ante las dificultades de la vida, gracias a la adecuada regulación de las emociones (Silva, 2005).

La regulación de las emociones resulta un elemento fundamental dentro del concepto de resiliencia (Troy $\&$ Mauss, 2011). En el momento de enfrentarse a situaciones estresantes, un adecuado control atencional junto a las habilidades necesarias para la reevaluación cognitiva permiten un procesamiento adaptativo de la información que terminará formando parte del acervo de respuestas almacenadas por el individuo ante situaciones críticas (figura 1). La neurociencia ha establecido claramente el sustrato neuronal responsable de este proceso. Estructuras del sistema límbico, como la amígdala, ajustan su funcionalidad a partir de la historia emocional del sujeto (Gordillo, Pérez, Arana, Mestas \& López, 2015), participando en la reevaluación como estrategia para la regulación de la experiencia emocional y sus componentes psicológicos y conductuales (Goldin, McRae, Ramel \& Gross, 2008). En definitiva, un adecuado procesamiento de las emociones tendría un efecto potencialmente beneficioso sobre las personas en el ámbito social, personal y clínico, con efectos a largo plazos reflejados en la capacidad de determinadas estructuras cerebrales para ajustarse a situaciones con una fuerte demanda emocional.

\section{¿Se puede aprender a gestionar mejor las emociones?}

En tanto la regulación emocional supone la habilidad para experienciar, reconocer y expresar las emociones de manera eficaz y con fluidez durante las interacciones sociales (Marans, Rubin \& Laurent, 2005), resulta frecuente que muchos trastornos mentales cursen con déficit en estas capacidades (Berking \& Wupperman, 2012). En este sentido, se ha planteado que la alta comorbilidad existente en los trastornos mentales podría explicarse en algún grado por los déficits en el procesamiento y la gestión de las emociones (Kret \& Ploeger, 2015). Esto parece claro si se tiene en cuenta que la mayoría de los trastornos mentales incluidos en el Manual diagnóstico y estadístico de los trastornos mentales (DSM-5; APA, 2013) cursan con déficit en la regulación emocional. Este es el caso de la depresión, con problemas en la identificación y modificación de las

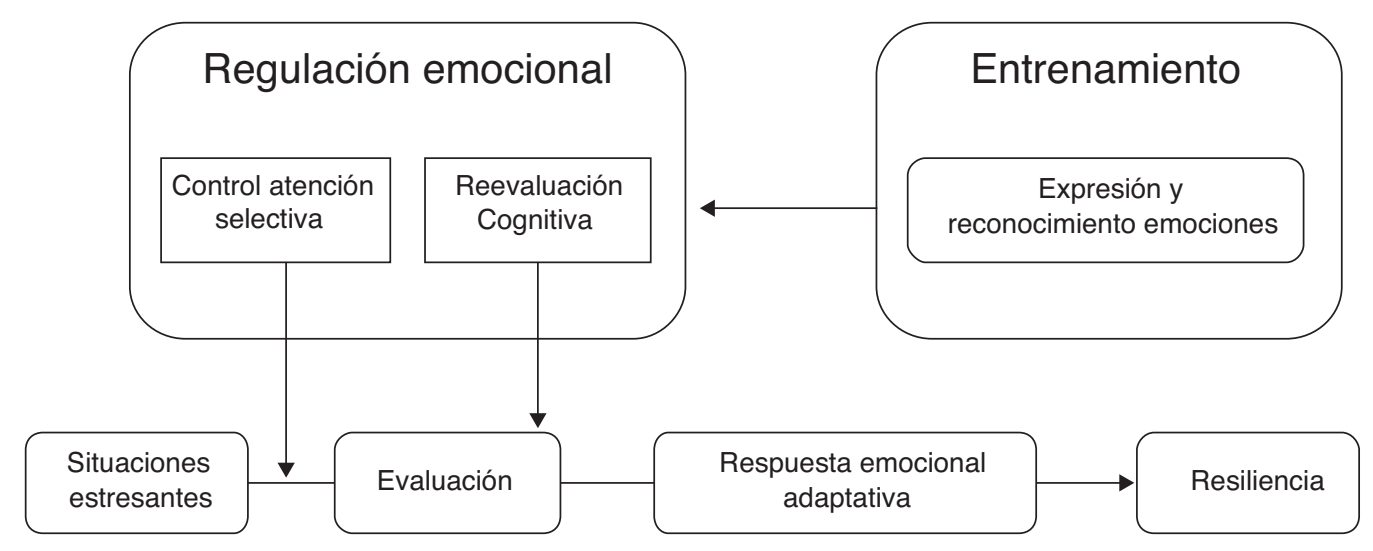

Figura 1. Modelo de la relación entre regulación emocional y resiliencia ante situaciones de estrés. Modificado de Troy y Gauss (2011). 
emociones y una baja tolerancia a las emociones negativas (Campbell-Sills, Barlow, Brown \& Hofmann, 2006; Rude \& McCarthy, 2003; Ehring, Fischer, Schnülle, Bösterling \& Tuschen-Caffier, 2008); la ansiedad, donde los déficits en la regulación emocional se han mostrado predictivos de la misma en la población no clínica, y se han asociado en la población clínica a una alta reactividad a las emociones negativas (Berking, Orth, Wupperman, Meier \& Caspar, 2008; CampbellSills, Ellard \& Barlow, 2014); los trastornos de la conducta alimentaria, con una intensidad incrementada de las experiencias emocionales (Svaldi, Griepenstroh, Tuschen-Caffier \& Ehring, 2012); las drogodependencias, donde el abuso de sustancias podría considerarse un medio de compensación ante la dificultad para regular las emociones negativas (p. ej., Wupperman et al., 2012); las psicopatologías infantiles (p. ej., trastorno de déficit de atención), con dificultades en el control emocional (Walcott \& Landau, 2004); la esquizofrenia, donde la preferencia por la utilización de estrategias de supresión emocional respecto a la reevaluación cognitiva genera problemas de ansiedad y adaptación social (Van der Meer, Van't Wout \& Aleman, 2009), y el autismo, donde las dificultades en la regulación emocional se han relacionado con incrementos en la ansiedad por la falta de habilidades con las que comprender las interacciones sociales (White, Mazefsky, Dichter, Chiu, Richey \& Ollendick, 2014).

Diferentes programas se han centrado en el tratamiento de la regulación emocional de manera específica para trastornos como la personalidad límite (Farrell \& Shaw, 1994), la anorexia (Money, Genders, Treasure, Schmidt \& Tchanturia, 2011), la ansiedad generalizada (Fresco, Mennin, Heimberg \& Ritter, 2013), las agresiones sexuales (Gillespie, Mitchell, Fisher \& Beech, 2012), el autismo (Mazefsky \& White, 2014). Otros programas más generales como el de Attwood (2004) (The Exploring Feelings Program), con un enfoque cognitivo-conductual y orientado al tratamiento de emociones concretas como la alegría, la relajación, la ira y la ansiedad. Mientras, las técnicas de mindfulness se enfocan a rebajar la reactividad emocional ante estresores vitales, por su importante valor a la hora de reducir la incidencia de determinados trastornos mentales (Britton, Shahar, Szepsenwol \& Jacobs, 2012). De igual manera, en neurorehabilitación empiezan a surgir estudios que implementan terapias para mejorar el estado afectivo del paciente, por sus implicaciones en la recuperación (Andrewes, Walter \& O’Neill, 2014). Por otro lado, las nuevas tecnologías permiten una metodología de entrenamiento virtual de la regulación emocional (Bosse, Gerritsen, De Man \& Treur, 2014), con un mantenimiento de los efectos a largo plazo que prometen su aplicabilidad a la población general en la ayuda para mejorar las capacidades de afrontamiento ante situaciones estresantes, o a poblaciones específicas como los cuerpos de seguridad del Estado, que suelen verse involucrados en situaciones donde deben ajustar sus emociones al contexto para mejorar la toma de decisiones.
Ahora bien, qué elementos se deben entrenar por ser los más eficaces. La regulación emocional depende en gran medida del grado de diferenciación emocional previo de la persona (Feldman, Gross, Conner \& Benvenuto, 2001). Es decir, depende de la capacidad para distinguir estados internos y diferenciarlos entre sí, y esto es debido a que, en tanto se es capaz de delimitar con mayor precisión la experiencia emocional, se puede manipular con mayor eficacia el estado de ánimo. Por lo tanto, la diferenciación emocional tiene un efecto significativo e inespecífico en la psicopatología por su papel en los procesos de regulación emocional (Silva, 2003). En otras palabras, cuanto más eficaces seamos a la hora de reconocer y expresar las emociones, con mayor facilidad podremos regularlas. De todo lo dicho se puede extraer la importancia de la expresión y comprensión emocional como vía de entrada a posibles terapias de entrenamiento en la mejora de la regulación emocional, bien porque esté presente un déficit asociado a otra patología, o bien dentro de la población normal para mejorar los procesos adaptativos ante situaciones que requieran un afrontamiento adecuado.

\section{Entrenando para reconocer y expresar las emociones}

Las habilidades sociales se ven moduladas por las capacidades en el reconocimiento y la expresión de las emociones. Es por esta razón por la que son muchos los programas orientados a incrementar las habilidades sociales en términos molares, que incluyen el tratamiento de las habilidades de reconocimiento emocional, como el entrenamiento en habilidades sociales (Bellack \& Hersen, 1979), o la terapia psicológica integrada (Roder, Brenner, Kienzle \& Hodel, 1992). Otros programas más precisos (moleculares) y centrados en la decodificación de las emociones, como el Micro-Expression Training Tool (METT; Ekman, 2002); el Emotion Training Program (ETP; Silver, Goodman, Knoll \& Isakov, 2004); el Training of Affect Recognition (TAR; Frommann, Streit \& Wölwer, 2003), o The Diagnostic Analysis of Nonverbal Accuracy 2 (DANVA 2; Nowicki \& Duke, 1994), que ha sido adaptado para su uso en la práctica clínica. De todos estos instrumentos se ha mostrado, en mayor o menor grado, su eficacia en la mejora de las habilidades de reconocimiento emocional en diferentes tipos de lesiones y trastornos mentales (p. ej., Russell, Chu \& Phillips, 2006; Radice-Newmann, Zupan, Tomita \& Willer, 2009; Wölwer, Frommann, Halfmann, Piaszek, Streit \& Gaebel, 2005).

El entrenamiento en aspectos concretos del procesamiento emocional empieza a ser una piedra de toque necesaria para entender la recuperación en diferentes patologías como las lesiones cerebrales, donde se ha comprobado la efectividad del entrenamiento en el reconocimiento de la expresión emocional (RadiceNewmann et al., 2009). Esta recuperación es posible por la capacidad de determinadas estructuras cerebrales, como el área premotora facial y el lóbulo parietal infe- 
rior, de compensar la funcionalidad cerebral en el procesamiento de las emociones ante lesiones de la amígdala (Becker et al., 2012). Por supuesto, esta capacidad compensatoria dependerá de la localización y gravedad de la lesión, pero resulta necesario una terapia de recuperación como base para favorecer este proceso compensatorio natural (Driscoll, Monte \& Grafman, 2011), y donde se integren los aspectos emocionales junto al resto de los procesos cognitivos (p. ej., Bayley et al., 2014).

De igual manera se puede decir respecto a otros trastornos, como la esquizofrenia, donde el adecuado procesamiento emocional es un factor determinante para la funcionalidad e independencia de este tipo de pacientes (Kimmy, Green, Mintz \& Brekke, 2003). Además, los déficits en el procesamiento de la información emocional en personas con riesgo de padecer esquizofrenia suponen un importante factor de vulnerabilidad (Phillips \& Seidman, 2008), que el entrenamiento emocional podría atenuar. Se puede decir lo mismo respecto a otro tipo de trastornos, como los de personalidad (p. ej., Domes, Schulze \& Herpertz, 2009); ansiedad, depresión y el estrés (Goldin \& Gross, 2010); o como el trastorno obsesivo compulsivo, donde se ha encontrado una reducción de los síntomas y una mejora en la calidad de vida de los pacientes (MacGuire et al., 2015). Si bien es cierto que la importancia terapéutica dada a la capacidad de reconocer y expresar correctamente las emociones está cada vez más aceptada, no es tanta la relevancia que se le da en el ámbito hospitalario como recurso de interacción con los pacientes.

\section{Las emociones en el contexto hospitalario}

La diferencia en el valor que el personal hospitalario y los pacientes dan a las emociones positivas se refleja en la importancia otorgada por el personal hospitalario y los pacientes de depresión a los aspectos más relevantes de la recuperación. En tanto los médicos consideran de mayor importancia el alivio de los síntomas depresivos, los enfermos están más preocupados por restaurar su afecto positivo (Demyttenaere et al., 2015a), con importantes consecuencias sobre la recuperación (Demyttenaere et al., 2015b). Basándonos en los trabajos consultados se puede decir que la capacidad para regular las emociones tiene una fuerte relación con la salud mental, y que las emociones positivas deberían centrar los esfuerzos de estos recursos regulatorios; es decir, dentro de un hospital hay que tener estrategias para afrontar las situaciones adversas que generan emociones negativas, pero también, al mismo tiempo, hay que promover la regulación de las emociones positivas, pero ¿cómo? Quizá esta «regulación positiva» pueda realizarse a través de la relación entre el personal sanitario y los pacientes.

Dentro del ámbito hospitalario, la empatía, como la capacidad de experimentar las emociones de los pacientes, ha demostrado ser un efectivo mecanismo que facilita la comunicación médico-paciente (Pollak et al., 2007), se asocia con una disminución de la ansiedad, un incremento de la satisfacción y una mejora de los resultados médicos (Hojat et al., 2011; Verheul, Sanders \& Bensing, 2010). Es uno de los procesos necesarios para poder detectar problemas emocionales en los pacientes y, por lo tanto, para poder actuar sobre ellos. Si bien ha resultado más habitual, una vez detectados los problemas emocionales, actuar sobre los déficits en la regulación de las emociones negativas, no ha sido hasta hace poco que se ha prestado interés a los beneficios de actuar sobre los déficits en la regulación de las emociones positivas (Carl, Soskin, Kerns \& Barlow, 2013). En tanto la gestión adecuada de las emociones positivas tendría beneficios importantes sobre el tratamiento (Ehrenreich, Fairholme, Buzzella, Ellard \& Barlow, 2007) y generaría grandes beneficios para la salud mental (Garland et al., 2010).

Uno de los problemas al tratar de regular las emociones positivas es que el placer es contingente con el cambio y desaparece con la satisfacción continua, pero esto no sucede con el displacer, que puede persistir en el tiempo si persisten las condiciones adversas (Frijda, 1998). En el contexto hospitalario, esto supone una lucha desigual en la que la persistencia de las condiciones contextuales negativas (las instalaciones hospitalarias) generaría el mantenimiento del afecto negativo, mientras el afecto positivo perduraría menor tiempo y requeriría de una sistemática administración de estimulación que permitiera niveles de afecto positivo perdurables en el tiempo. Este refuerzo sobre la regulación de las emociones positivas se ha mostrado efectivo en la recuperación de diferentes trastornos como las adicciones (Ardebili, Afkari, Ghasemi, Dastoorpour \& Ghasem, 2013), mientras que la falta de refuerzo positivo o un exceso en el refuerzo de las conductas depresivas de los hijos se relacionan con la depresión infantil (Schwartz, Séller, Dudgeon \& Allem, 2012).

Precisamente, el contexto hospitalario supone una de las claves contextuales aversivas más implicadas en el mantenimiento del estado afectivo negativo y poco favorable al mantenimiento de las emociones positivas. Si bien las evidencias parecen claras respecto a la necesidad de regular este desequilibrio, ¿qué puede hacer el personal sanitario al respecto? Es decir, ¿se puede sistematizar la regulación emocional del enfermo a través de la comunicación entre el personal sanitario y el paciente? Las evidencias más claras de los beneficios de la intervención sobre los procesos emocionales se han obtenido de la población clínica, pero cabe pensar que la población con problemas de salud puntuales, o bien en la fase previa al diagnóstico de una enfermedad o trastorno mental, o la simple consulta con el especialista, se beneficie igualmente de un contexto hospitalario adecuado a nivel emocional, donde el personal sanitario jugaría un papel fundamental.

Por otro lado, la literatura reciente manifiesta la necesidad de entrenar al personal hospitalario en su relación con el contexto clínico, en tanto un alto porcentaje, según Berkow Virkstis, Stewart y Conway 
(2009) del 90\%, inicia su trabajo sin estar bien preparada para la práctica clínica. Por lo tanto, el contacto directo con el ambiente que se vive en el día a día hospitalario resultaría un factor determinante para evitar problemas en la praxis clínica (Ayers et al., 2015) y una mejor adaptación emocional del personal hospitalario que redundaría en una interacción más efectiva con el enfermo. Esta interacción con el enfermo conlleva beneficios que han sido establecidos de manera muy clara en la literatura científica. La sonrisa que se genera de manera reactiva como respuesta a una sensación de felicidad tiene efectos positivos sobre el bienestar de las personas y negativos cuando se produce de manera frecuente y proactiva; es decir, cuando se utiliza para enmascarar una emoción negativa (p. ej., ocultar tristeza o miedo ante la enfermedad) o llegar a un estado afectivo positivo (Labroo, Mukhopadhyay \& Dong, 2014). Este efecto se debe a la interpretación que hace el emisor del origen de su propia sonrisa, pero también de la sonrisa de los demás. Al mismo tiempo, el contacto físico (p. ej., tocar el hombro mientras se sonríe) jugaría un papel importante al incrementar la atención sobre la emoción expresada y sobre lo agradable de la persona que sonríe (Ellingsen, Wessberg, Chelnokova, Olausson, Laeng \& Leknes, 2014). En el ámbito clínico, esto puede resultar adecuado para conectar emocionalmente con los pacientes que, en muchas ocasiones, están centrados en sus problemas de salud. Pero deben tenerse en cuenta otros factores que puedan hacer que el contacto físico sea contraproducente, como podría ser la personalidad del paciente (p. ej., una persona introvertida podría percibir como negativo el contacto físico), el nivel de ansiedad o las características de la enfermedad.

Por otro lado, en estados de ansiedad muy habituales en el ámbito hospitalario, las personas generan sesgos hacia determinados tipos de expresiones (Tran, Lamplmayr, Pintzinger \& Pfabigan, 2013). Esto resulta de gran importancia dado que no solo se producen sesgos hacia determinadas expresiones como la ira o la tristeza, sino que también conlleva una interpretación sobre las causas de dicha expresión. Una interpretación inadecuada de una expresión facial podría generar estados afectivos negativos no controlados con efectos perjudiciales sobre la recuperación del paciente.

\section{Conclusiones}

Este campo de investigación requiere de un análisis en profundidad para establecer protocolos emocionales de interacción que no estarían exentos de dificultades, dado que también habría que tener en cuenta el estado afectivo del personal sanitario. La alta prevalencia del síndrome de burnout en estos profesionales podría inhibir la implantación de un protocolo eficaz, puesto que no se pueden ajustar pautas de expresividad emocional positivas bajo un estado de cansancio, ansiedad o estrés. Para abordar este problema se deben integrar tanto los aspectos emocionales del paciente como los del personal sanitario en la búsqueda de un contexto de interacción positivo a nivel emocional que procure beneficios clínicos.

De esta aproximación teórica al problema se pueden concluir los siguientes puntos: a) el afecto positivo se relaciona con una mayor salud física y mental, y una mejor calidad de vida; b) la resiliencia y la regulación emocional son dos variables relevantes que podrían estar mediando en esta relación; c) se puede entrenar en la regulación de las emociones, tanto en la población clínica como en la no clínica, con beneficios en el tratamiento y la prevención respectivamente; d) el entrenamiento en el reconocimiento y la expresión de las emociones es uno de los elementos más eficaces y de fácil aplicación en terapias específicas para diferentes trastornos mentales; e) una forma de entrenar en la regulación emocional, con un alto poder ecológico, es potenciar la interacción emocional eficiente entre el personal sanitario y los pacientes, a través de la adecuada capacitación en el reconocimiento y la expresión de las emociones del personal sanitario, mejorando la empatía, y con importantes beneficios sobre las praxis y eficacia terapéutica.

\section{Referencias}

American Psychiatric Association. (2013). Diagnostic and statistical manual of mental disorders (5th ed.). Washington, DC: Author.

Andrewes, E. H., Walter, V. \& O’Neill, B. (2014). Exploring the use of positive psychology interventions in brain injury survivors with challenging behaviour. Brain Injury, 28(7), 965-971. DOI: 10.3109/02699052. 2014.888764

Ardebili, M. E., Afkari, M. E., Ghasemi, F., Dastoorpour, M. \& Ghasem, A. (2013). The Effect of Empowerment on the Reinforcement of Positive Emotions in Methamphetamine Addicts. Middle-East Journal of Scientific Research, 15(2), 272-277, 2013. DOI: 10.5829/ idosi.mejsr.2013.15.2.653

Attwood, T. (2004). Exploring Feelings. Arlington TX: Future Horizons.

Ayers, C. J., Binder, B. K., Lyon, J. C., Montgomery, D., Joci, A. \& Foster, W. A. (2015). The simulated hospital environment: A qualitative study applying space industry techniques. Journal of Professional Nursing, 31(1), 18-25. DOI: 10.1016/j.profnurs.2014.06.002

Bayley, M. Th., Tate, R., Douglas, J. M., Turkstra, L. S., Ponsford, J., Stergiou-Kita, M., Kua, A. \& Bragge, P. (2014). INCOG Guidelines for Cognitive Rehabilitation Following Traumatic Brain Injury: Methods and Overview. Journal of head trauma Rehabilitation, 29(4), 290-306. doi: 10.1097/HTR.0000000000000070

Becker, B., Mihov, Y., Scheele, D., Kendrick, K. M., Feinstein, J. S., Matusch, A., Aydin, M., Reich, H., Urbach, H., Oros-Peusquens, A-M., Shah, N. J., Kunz, W. S., Schlaepfer, T. E., Zilles, K., Maier, W. \& Hurlemann, R. (2012). Fear processing and social networking in the absence of a functional amygdala. Biological Psychiatry, 72(1), 70-77. DOI: 10.1016/j.biopsych.2011.11.024 
Bellack, A. S. \& Hersen, M. (1979). Research and practice in social skills training. Springer-Verlag US 1979. 10.

Berkow, S., Virkstis, K., Stewart, J. \& Conway, L. (2009). Assessing new graduate nurse performance. Nurse Educator, 34, 17-22. DOI: 10.1097/01.NNE.00003434 05.90362 .15

Berking, M. \& Wupperman, P. (2012). Emotion regulation and mental health: recent findings, current challenges, and future directions. Current Opinion in Psychiatry, 25(2), 128-134. DOI: 10.1097/YCO.0b013e 3283503669

Berking, M., Orth, U., Wupperman, P., Meier, L. \& Caspar, F. (2008). Prospective effects of emotion regulation on emotional adjustment. Journal of Counseling Psychology, 55(4), 485-494. DOI: doi: 10.1037/ a0013589

Berking, M. \& Wupperman, P. (2012). Emotion regulation and mental health: recent findings, current challenges and future directions. Current Opinion in Psychiatry, 25(2), 128-134. DOI: 10.1097/YCO.0b013e 3283503669

Bosse, T., Gerritsen, C., de Man, J. \& Treur, J. (2014). Towards virtual training of emotion regulation. Brain Informatics, 1(1-4), 27-37. DOI: 10.1007/s40708014-0004-9

Britton, W. B., Shahar, B., Szepsenwol, O. \& Jacobs, W. J. (2012). Mindfulness-Based cognitive therapy improves emotional reactivity to social stress: Results from a randomized controlled trial. Behavior Therapy, 43(2), 365-380. DOI: 10.1016/j.beth.2011.08.006.

Cameron, D. S., Bertenshaw, E. M. \& Sheeran, P. (2014). The impact of positive affect on health cognitions and behaviours: A meta-analysis of the experimental evidence. Health Psychology Review. DOI: 10.1080/ 17437199.2014.923164.

Campbell-Sills, L., Barlow, D. H., Brown, T. A. \& Hofmann, S. G. (2006). Effects of suppression and acceptance on emotional responses of individuals with anxiety and mood disorders. Behaviour Research and Therapy, 44, 1251-1263. DOI: 10.1016/j.brat.2005.10.001

Campbell-Sills, L., Ellard, K. \& Barlow, D. H. (2014). Emotion regulation in anxiety disorders. En J. J. Gross (Ed.), Handbook of emotion regulation (2nd ed., pp. 393-412). New York, NY: Guilford Press.

Carl, J. R., Soskin, D. P., Kerns, C. \& Barlow, D. H. (2013). Positive emotion regulation in emotional disorders: A theoretical review. Clinical Psychology Review, 33(3), 343-360. DOI: 10.1016/j.cpr.2013.01.003

Carretero, R. (2010). Resiliencia. Una visión positiva para la prevención e intervención desde los servicios sociales. Nómadas, 27. Recuperado de http://www. redalyc.org/articulo.oa?id=18113757004.

Demyttenaere, K., Donneau, A-F., Albert, A., Ansseau, M., Constant, E. \& Heeringen, F. (2015a). What is important in being cured from depression? Discordance between physicians and patients (1). Journal of Affect Disorders, 174, 390-396. DOI: 10.1016/j. jad.2014.12.004.

Demyttenaere, K., Donneau, A-F., Albert, A., Ansseau, M., Constant, E. \& Heeringen, F. (2015b). What is important in being cured from: Does discordance between physicians and patients matter? (2). Journal of Affect Disorders, 174, 390-396. DOI: 10.1016/j. jad.2014.12.002

Domes, G., Schulze, L. \& Herpertz, S. C. (2009). Emotion recognition in borderline personality disorden: A review of the literature. Journal of Personality Disorders, 23(1), 6-19. DOI: 10.1521/pedi.2009.23.1.6

Driscoll, D. M., Monte, O. D. \& Grafman, J. (2011). A need for improved training interventions for the remediation of impairments in social functioning following brain injury. Journal of Neurotrauma, 28(2): 319-326. DOI: 10.1089/neu.2010.1523.

Ekman, P. (2002). MicroExpression Training Tool (METT). San Francisco: University of California.

Ellingsen, D-M., Wessberg, J., Chelnokova, O., Olausson, H., Laeng, B. \& Leknes, S. (2014). In touch with your emotions: Oxytocin and touch change social impressions while others' facial expressions can alter touch. Psychoneuroendocrinology, 39, 11-20. DOI: 10.1016/j.psyneuen.2013.09.017.

Ehrenreich, J. T., Fairholme, C. P., Buzzella, B. A., Ellard, K. K. \& Barlow, D. H. (2007). The role of emotion in psychological therapy. Clinical Psychology: Science and Practice, 14(4), 422-428. DOI: 10.1111/j.1468-2850. 2007.00102.x.

Ehring, T., Fischer, S., Schnülle, J., Bösterling, A. \& Tuschen-Caffier, B. (2008). Characteristics of emotion regulation in recovered depressed versus never depressed individuals. Personality and Individual Differences, 44(7), 1574-1584. DOI: 10.1016/j.paid.2008. 01.013

Farrell, J. \& Shaw, I. A. (1994). Emotional awareness training: A prerequisite to effective cognitive-behavioral treatment of borderline personality disorder. Cognitive and Behavioral Practice, 1(1), 71-91. DOI: 10. 1016/S1077-7229(05)80087-2

Feldman, L. B., Gross, J. J., Conner, T. \& Benvenuto, M. (2001). Knowing what you're feeling and knowing what to do about it: mapping the relation between emotion differentiation and emotion regulation. Cognition and Emotion, 15(6), 713-724. DOI: 10.1080/ 02699930143000239

Frommann, N., Streit, M. \& Wölwer, W. (2003). Remediation of facial affect recognition impairments in patients with schizophrenia: A new training program. Psychiatry Research, 117(3), 281-284. DOI: 10.1093/ schbul/sbr071

Fresco, D. M., Mennin, D. S., Heimberg, R. G. \& Ritter, $M$ (2013). Emotion regulation therapy for generalized anxiety disorder. Cognitive and Behavioral Practice, 20(3), 282-300. DOI: 1077-7229/13/282-300\$1.00/0

Frijda, N. H. (1998). The laws of emotion. American Psychologist, 43, 349-358. doi: 10.1037/0003-066X.43. 5.349

Garland, E. L., Fredrickson, B., Kring, A. M., Johnson, D. P., Meyer, P. S. \& Penn, D. L. (2010). Upward spirals of positive emotions counter downward spirals of negativity: Insights from the broaden-and-build theory and affective neuroscience on the treatment 
of emotion dysfunctions and deficits in psychopathology. Clinical Psychology Review, 30(7), 849-864. DOI: 10.1016/j.cpr.2010.03.002

Gillespie, S. M., Mitchell, I. J., Fisher, D. \& Beech, A. R., (2012). Treating disturbed emotional regulation in sexual offenders: The potential applications of mindful self-regulation and controlled breathing techniques. Aggression and Violent Behavior, 17(4), 333-343. DOI: 10.1016/j.avb.2012.03.005

Goldin, P. R. \& Gross, J. J. (2010). Effects of mindfulness-based stress reduction (MBSR) on emotion regulation in social anxiety disorder. Emotion, 10(1), 83-91. DOI: 10.1037/a0018441

Goldin, P. R., McRae, K., Ramel, W. \& Gross, J. J. (2008). The neural bases of emotion regulation: reappraisal and suppression of negative emotion. Biological Psychiatry, 63, 577-586. DOI: 10.1016/j.biopsych.2007. 05.031

Gordillo, F., Pérez, M. A., Arana, J. M., Mestas L. \& López, R. M. (2015). Efecto de la experiencia en la neurología de la expresión facial. Revista de Neurología, 60(7), 316-320.

Hojat, M., Louis, D. Z., Markham, F. W., Wender, R., Rabinowitz, C. \& Gonnella, J. S. (2011). Physicians' empathy and clinical outcomes for diabetic patients. Academic Medicine, 86(3), 359-364. DOI: 10.1097/ ACM.0b013e3182086fe1

Hu, T., Zhang, D. \& Wang, J. (2015). A meta-analysis of the trait resilience and mental health. Personality and Individual Differences 76, 18-27. DOI: 10.1016/j. paid.2014.11.039

Kimmy, K. S., Green, M. F., Mintz, J. \& Brekke, J. S. (2003). Is emotion processing a predictor of functional outcome in schizophrenia? Schizophrenia Bulletin, 29(3), 487-497.

Kret, M. E. \& Ploeger, A. (2015). Emotion processing deficits: A liability spectrum providing insight into comorbidity of mental disorders. Neuroscience and Biobehavioral Reviews, 52, 153-171. DOI: 10.1016/j. neubiorev.2015.02.011

Lyubomirsky, S., King, L. \& Diener, E. (2005). The Benefits of Frequent Positive Affect: Does Happiness Lead to Success? Psychological Bulletin, 131(6), 803855. DOI: 0.1037/0033-2909.131.6.803.

Labroo, A. A., Mukhopadhyay, A. \& Dong, P. (2014). Not always the best medicine: Why frequent smiling can reduce wellbeing. Journal of Experimental Social Psychology, 53, 156-162. DOI: 10.1016/j.jesp.2014.03. 001.

MacGuire, J. F., Arnold, E., Park, J. M., Nadeau, J. M., Lewin, A. B., Murphy, T. K. \& Storch, E. A. (2015). Living with tics: Reduced impairment and improved quality of life for youth with chronic tic disorders. Psychiatry Research, 225(3), 571-579. DOI: 10.1016/j. psychres.2014.11.045

Marans, W. D., Rubin, E. \& Laurent, A. (2005). Addressing social communication skills in individuals with high functioning autism and Asperger Syndrome: Critical priorities in educational programming. En: ER. Volkmar, A. Klin, \& R. Paul (Eds.), Handbook of autism and pervasive developmental disorders (3rd ed.). New York: Wuey.

Mazefsky, C. A. \& White, S. W. (2014). Emotion regulation concepts \& practice in autism spectrum disorder. Child \& Adolescent Psychiatric Clinics of North America, 23(1), 15-24. DOI: 10.1016/j.chc.2013.07.002 Money, C., Genders, R., Treasure, J., Schmidt, U. \& Tchanturia, K. (2011). A brief emotion focused intervention for inpatients with anorexia nervosa: A qualitative study. Journal of Health Psychology, 16(6), 947-958. DOI: 10.1177/1359105310396395

Nowicki, S. J., Jr. \& Duke, M. P. (1994). Individual differences in the nonverbal communication of affect: The diagnostic analysis of nonverbal accuracy scale. Journal of Nonverbal Behavior, 18(1), 9-35. DOI: 10.1007/BF02169077

Phillips, L. K. \& Seidman, L. J. (2008). Emotion processing in person at Risk for Schizophrenia. Schizophrenia Bulletin, 34(5), 888-903. DOI: 10.1093/schbul/ sbn085

Pollak, K. I., Arnold, R. M., Jeffreys, A. S., Alexander, S. C., Olsen, M. K., Abernethy, A. P., Skinner, C. S., Rodríguez, K. L. \& Tulski, J. A. (2007). Oncologist communication about emotion during visits with patients with advanced cancer. Journal of Clinical Onclogy, 25(36), 5748-5752. DOI: 10.1200/ JCO.2007.12.4180

Radice-Neumann, D., Zupan, B., Tomita, M. \& Willer, B. (2009). Training emotional processing in person with brain injury. The Journal of Head Trauma Rehabilitation, 24(5), 313-123. DOI: 10.1097/HTR.0b013e 3181 b09160

Roder, V., Brenner, H. D., Kienzle, N. \& Hodel, B. (1992). Integriertes psychologisches therapieprogramm für schizophrene patienten (IPT). Psychologie Verlagsunion, Weinheim.

Rude, S. S. \& McCarthy, C. T. (2003). Emotional functioning in depressed and depression vulnerable college students. Cognition and Emotion, 17(5), 799806. DOI: 10.1080/02699930302283

Schwartz, O. S., Séller, L. B., Dudgeon, P. \& Allem, N. B. (2012). Emotion socialization within the family environment and adolescent depression. Clinical Psychology Review 32(6), 447-453. DOI: 10.1016/j. cpr.2012.05.002

Russell, T. A., Chu, E. \& Phillips, M. L. (2006). A pilot study to investigate the effectiveness of emotion recognition remediation in schizophrenia using the micro-expression training tool. British Journal of Clinical Psychology, 45 (Pt 4), 579-583. DOI: 10.1348/ $014466505 \times 90866$

Silva, J. (2003). Biología de la regulación emocional: su impacto en la psicología del afecto y la psicoterapia. Terapia Psicológica, 22(1), 163-172.

Silva, J. (2005). Regulación emocional y psicopatología: el modelo de vulnerabilidad/resiliencia. Revista Chilena de Neuropsiquiatría, 43(3), 201-209.

Silver, H., Goodman, C., Knoll, G. \& Isakov, V. (2004). Brief emotion training improves recognition of facial emotions in chronic schizophrenia. A pilot study. 
Psychiatry Research, 128(2), 147-154. DOI: 10.1016/j. psychres.2004.06.002

Svaldi, J., Griepenstroh, J., Tuschen-Caffier, B. \& Ehring, T. (2012). Emotion regulation deficits in eating disorders: A marker of eating pathology or general psychopathology? Psychiatry Research, 197(1), 103111. DOI: 10.1016/j.psychres.2011.11.009

Tran, U. S., Lamplmayr, E., Pintzinger, N. M. \& Pfabigan, D. M. (2013). Happy and angry faces: Subclinical levels of anxiety are differentially related to attentional biases in men and women. Journal of Research in Personality, 47(4), 390-7. DOI: 10.1016/j. jrp.2013.03.007

Troy, A. S. \& Mauss, I. B., (2011). Resilence in the face of stress: emotion regulation as a protective factor. En Resilience and Mental Health: Challenges Across the Lifespan, ed. Steven M. Southwick, Brett T. Litz, Dennis Charney, and Matthew J. Friedman. Published by Cambridge University Press.

Van der Meer, L., Van't Wout, M. \& Aleman, A. (2009). Emotion regulation strategies in patients with schizophrenia. Psychiatry Research, 170(2-3), 108-113. DOI: $10.1016 /$ j.psychres.2009.07.010

Vera, B. (2006) Psicología positiva: una nueva forma de entender la psicología. Papeles del Psicólogo, 27, 3-8.

Verheul, W., Sanders, A. \& Bensing, J. (2010). The effects of physicians' affect-oriented communication style and raising expectations on analogue patients' anxie- ty, affect and expectancies. Patient Education and Counseling, 80(3), 300-306. DOI: 10.1016/j.pec.2010. 06.017

Walcott, C. M. \& Landau, S. (2004). The relation between disinhibition and emotion regulation in boys with attention deficit hyperactivity disorder. Journal of Clinical Child and Adolescent Psychology, 33(4), 772782. DOI: $10.1207 / \mathrm{s} 15374424$ jccp3304_12

White, S. W., Mazefsky, C. A., Dichter, G. S., Chiu, P. H., Richey, J. A. \& Ollendick, T. H. (2014). Socialcognitive, physiological, and neural mechanisms underlying emotion regulation impairments: understanding anxiety in autism spectrum disorder. International Journal of Developmental Neuroscience 39, 2-36. DOI: 10.1016/j.ijdevneu.2014.05.012

Wölwer, W., Frommann, N., Halfmann, S., Piaszek, A., Streit, M. \& Gaebel, W. (2005). Remediation of impairments in facial affect recognition in schizophrenia: Efficacy and specificity of a new training program. Schizophrenia Research 80(2-3), 295-303. DOI: $10.1016 / \mathrm{S}$

Wupperman, P., Marlatt, G. A., Cunningham, A., Bown, S., Berking, M., Mulvihill-Rivera, N. \& Easton, C. (2012). Mindfulness and modifi cation therapy for behavior dysregulation: Results from a pilot study targeting alcohol use and aggression in women. Journal of Clinical Psychology, 68(1), 50-66. DOI: 10.1002/jclp. 20830 